\title{
Dural AVFS
}

\section{Clinical Presentation, Classification and Angiograpic Evaluation}

\author{
K.G. TERBRUGGE \\ University Health Network; University of Toronto; Canada
}

\section{Definition}

Dural arteriovenous fistulas are abnormal vascular communications within the dura fed by enlarged dural arteries and with early drainage into dural sinuses or cortical veins. Although the term vascular malformation implies a congenital origin, most the lesions are acquired, and related to a previous episode of infection, trauma, haemorrhage or previous surgical intervention.

\section{Classification}

Dural arteriovenous fistulas can be classified according to their location, however, the most important aspect of the lesion from a clinical point of view is the presence or absence of cortical venous drainage. Certain locations (tentorial and anterior cranial fossa) are more commonly associated with cortical venous drainage than others.

\section{Clinical Presentation}

The symptomatology of dural AVFs is related to the turbulence of blood in the nidus and the venous drainage pattern. A bruit is probably the most common presentation, but other symptoms may include headaches, cranial nerve palsies, focal central nervous system deficit, seizures, haemorrhage, ventricular enlargement, and congenital heart failure.

The bruit is caused by the turbulence of

blood in the nidus adjacent to the petrous bone or venous drainage towards that level. High flow lesions in particular in the pediatric age group may give rise to congestive heart failure. Sometimes, the venous outflow channel may become significantly enlarged, and in these cases may cause local mass effect. Increased venous pressure may lead to decreased cerebrospinal fluid absorption and ventricular enlargement or to increased intracranial pressure and papilledema. Drainage into cortical cerebral veins will cause congestion of the drainage of the brain itself in that area or even remote from that area and cause secondary CNS symptoms, such as neurological deficit, seizures and intracranial haemorrhage.

Dural arteriovenous fistulas located along the anterior cranial fossa and tentorium are almost always associated with cortical venous drainage, and their most common presentation is haemorrhage (SAH, $\mathrm{ICH}$, and $\mathrm{SDH}$ ).

Dural arteriovenous malformations along the torcular and lateral sinuses most commonly present with a bruit and headaches. CNS symptomatology may be present when the lesion is associated with cortical venous drainage. Clinical symptoms may disappear or change and this may indicate a change in the venous outflow pattern. Hormonal influences upon the symptomatology are known to occur.

The natural history of the disease is not completely understood. Spontaneous regression of the dural AVF is known to occur, in particular 
in slow flow lesions. Regression is sometimes induced by angiography or intermittent compression of the feeding vessels. Progressive enlargement of the lesion has been described following pregnancy. Spontaneous transformation of a lesion with sinosal drainage into one with cortical venous drainage appears extremely rare.

\section{Radiological Investigation}

Plain skull films are generally normal, although enlarged skull foramina related to the increased size of the feeding arteries and draining veins can sometimes be identified. $\mathrm{CT}$ and MR findings are limited to the demonstration of possible ventricular enlargement or the demonstration of prominent draining veins. The MRI appearance of the secondary effect of passive venous congestion on the affected brain reveals evidence of brain edema in the presence of prominent vascular channels on the surface of the brain. Haemorrhagic complications are best shown by CT and MRI, but most of the time the CT and MRI examinations will not disclose the underlying pathology.

Angiography should be done using a superselective intra-arterial approach. A protocol approach is recommended. Special attention should be paid to the complete study of all the feeding arteries, the angio-architecture of the nidus (vascular network versus fistula), the venous drainage pattern of the fistula as well as the brain, and the presence of other venous abnormalities such as thrombosis.

\section{Treatment}

Because dural arteriovenous fistulas in most instances represent a benign disease, treatment should be conducted with this mind. In general, however, treatment is indicated with cortical venous drainage is noted to be present. Treatment should also be performed whenever systemic symptoms are present, such as congestive heart failure, and in patients with raised intracranial pressure. Occasionally, the presence of bruit can become incompatible with the patient's normal functioning, and present a subjective indication to treatment.

The treatment can be embolisation or surgery, or a combination of both. As a general principle, the aggressiveness of the treatment will depend on the presence of leptomeningeal venous drainage (cortical reflux), and the clini- cal symptomatology. With respect to embolisation, this means that particle embolisation is performed for those patients with subjective symptoms and sinosal venous drainage, while a combination of particle embolisation and NBCA is used for those patients who have a dural arteriovenous malformation with cortical venous drainage. The results of particle embolisation are frequently not stable, and repeated embolisations may be necessary while the results of NBCA embolisation are more likely to be stable. If transarterial embolisation proves to be not feasible, or not successful, then one might consider either the venous approach, or intra-operative embolisation. If the venous approach is to be considered, then careful analysis of the venous drainage patterns of the normal brain is to be performed in order to assess whether the venous outflow segment of the dural AVM can be safely sacrificed (balloons, coils, etc.). Intra-operative injection of NBCA into the nidus of the lesion with temporary venous outflow clipping has been performed in dural AVMs with deep midline venous drainage patterns at the level of the midbrain and tentorium, with promising results. For those patients who presented with CNS symptoms, and in whom embolisation proved to be incomplete, surgical or disconnection of the venous communication to the cortical venous system of the brain should be performed. The role of stereotactic radiosurgery is still under investigation.

\section{References}

1 Lasjaunias P, Chiu M: et Al: Spontaneous intracranial dural arteriovenous malformations, neurological manifestations. J Neurosurg 67: 724-730, 1986.

2 Satomi J, van Dijk JMC et Al: Benign cranial dural arteriovenous fistulas: Outcome of conservative management based on the natural history. J Neurosurgery 97 : 767-770, 2003.

3 Satomi J, vanDijk M et Al: Benign Cranial Dural Arteriovenous Fistulas: Natural History and Proposed Management. Journal of Neurosurgery 97: 749-750, 2002.

4 VanDijk M, terBrugge K et Al: The Clinical Course of Cranial Dural AV-Fistulas with Long -term Persistent Cortical Venous Reflux. Stroke 33: 1233-1236, 2002.

5 Lasjaunias P, Berenstein A, terBrugge K: Surgical Neuroangiography, Volume $2 \mathrm{Ch} 8$. Dural Arteriovenous Shunts Springer-Verlag publishers, 2003.

Karel Gerard terBrugge, M.D. Western Hospital

Diag \& Therap Neuroradiology

399 Bathurst Street

CND Toronto Ontario M5T 2S8; Canada 